\title{
Arterial injury during transcatheter arterial chemoembolization for hepatocellular carcinoma: predictors of risk and outcome
}

Hironori Onizuka, MD, Eijun Sueyoshi, MD, Hideki Ishimaru, MD, Ichiro Sakamoto, MD, Masataka Uetani, MD

Department of Radiology, Nagasaki University School of Medicine

Corresponding author: Eijun Sueyoshi, MD

Department of Radiology, Nagasaki University School of Medicine, 1-7-1

Sakamoto, Nagasaki 852-8501, Japan

Tel.: +81-95-849-7354; Fax: +81-95-849-7357

E-mail: sueyo@nagasaki-u.ac.jp

Authors' e-mail addresses

Hironori Onizuka: dream_match_never_ends@yahoo.co.jp

Hideki Ishimaru: ishi_maru@yahoo.co.jp

Ichiro Sakamoto: icsakamoto@yahoo.co.jp

Masataka Uetani: uetani@nagasaki-u.ac.jp

Manuscript Type : Clinical investigation

Conflict of Interest: The authors declare that they have no conflict of interest 
Objective: To assess clinical features and results of follow-up in patients with arterial injury during transcatheter arterial chemoembolization (TACE) for hepatocellular carcinoma.

Materials and methods: From February 2005 to December 2015, 2,219 TACE procedures were performed in 906 patients at our hospital. latrogenic arterial injury occurred during 38 TACE procedures (sessions) in 35 patients (24 men, 11 women; mean age 71.8 years, range 37-92 years). The incidence of arterial injury was $1.7 \%$. We evaluated characteristics of arterial injury, and evaluated the risk factors for incomplete recanalization of the injured artery at follow-up angiography conducted after 1-11 months (mean 102.5 days).

Results: latrogenic arterial injury was caused by the microcatheter in 34 of 38 cases $(86.8 \%)$. There were 15 cases $(39.5 \%)$ in which the replaced hepatic artery or the extrahepatic artery was the parasitic supply. Extravasation occurred in five cases. We divided 36 cases into two groups: complete reopening $(n=24)$ and non-complete reopening $(n=12)$. The two groups were compared regarding the factors associated with incomplete recanalization of the injured artery at follow-up. Injury length $>3 \mathrm{~cm}(p=0.0002)$ and proximal arterial injury (proximal to the segmental artery; $p=0.03$ ) were significant risk factors for non-complete reopening of the injured artery.

Conclusion: latrogenic arterial injury frequently occurred in the extrahepatic artery or replaced hepatic artery. Recanalization rate of arterial injury was high; however, injury length $>3 \mathrm{~cm}$ and proximal arterial injury were risk factors for non-complete reopening of the injured artery. 


\section{Introduction}

Transcatheter arterial chemoembolization (TACE) is widely used in the management of hepatocellular carcinoma (HCC). $\mathrm{HCC}$ chemoembolization is based on the fact that the normal liver parenchyma receives a dual blood supply from the hepatic artery and the portal vein, whereas HCCs are exclusively supplied by the hepatic artery [1, 2]. Recently, TACE has been indicated for the treatment of intermediate-stage $\mathrm{HCC}[3,4]$.

Unexpected iatrogenic arterial injury has been reported during selective catheterization for TACE $[3,6,7]$. However, to our knowledge, no studies have reported on TACE procedures using microcatheters. In addition, with recent developments in the TACE technique, the number of cases undergoing repeated TACE has increased, and the use of TACE through extrahepatic collateral vessels has also increased.

We evaluated clinical features and results of follow-up in patients with iatrogenic arterial injury during TACE for HCC. The purpose of this study was to identify predictors for arterial injury, as well as the likelihood of recanalization as assessed in a subsequent follow-up procedure.

\section{Materials and Methods}

Patients

The ethics committee of our hospital approved this study and waived informed consent. From February 2005 to December 2015, 2,219 TACE sessions were performed in 906 patients at our hospital. We excluded cases of iatrogenic arterial injury from the femoral artery to the aorta during puncture and sheath 
insertion. latrogenic arterial injury occurred in 38 cases (TACE sessions) in 35

patients. The incidence of arterial injury was $1.7 \%$.

The 35 patients included 24 men and 11 women, aged 37-92 years (mean 71.8 years). The demographic characteristics and clinical profiles of the 38 cases are shown in Table 1.

\section{TACE procedure}

All TACE procedures were performed using digital subtraction angiography (DSA) with the contrast medium iopamidol 300 (Iopamiron 300; Schering, Bonn, Germany) or iomeprol 300 (Iomeron 300; Bracco, Milan, Italy). TACE was performed with a 4-F catheter for celiac and superior mesenteric arteriography, and/or for inferior phrenic arteriography.

Microcatheters (Progreat; Terumo, Tokyo, Japan or PIXIE; Tokai Medical Products, Aichi, Japan) were coaxially inserted into a 4-F catheter as selectively as possible through the lobar, segmental, or subsegmental arteries, depending on the tumor distribution and hepatic functional reserve. A 0.035 -inch steerable guidewire (Terumo Co., Tokyo, Japan) was used in all patients. The microcatheter was inserted into the distal portion using a 0.016 -inch guide wire (GT Wire; Terumo, Tokyo, Japan or Meister; Asahi Intecc Co. Ltd., Tokyo, Japan) with approximately $1 \mathrm{~cm}$ of the tip bent into a $\mathrm{J}$ shape.

Initially, an emulsion of 2-10 mL lipiodol (Lipiodol Ultrafluide; Laboratoire Guerbet, Aulnay-Sous-Bois, France), 20-50 mg doxorubicin hydrochloride, and 2-10 mg mitomycin- $\mathrm{C}$ was administered into the feeder vessels. The dosage of lipiodol, doxorubicin, and mitomycin-C was determined according to tumor size, 
vascularity, presence of arterioportal shunt, and underlying liver function. Next, gelatin sponge particles mixed with contrast material were administered into the feeder vessels until stasis of arterial flow was achieved.

Follow-up angiography

In 36 of 38 cases (94.7\%), follow-up angiography (TACE or transcatheter arterial infusion) was performed within 1 year. The time interval between arterial injury and the first follow-up angiography ranged from 1-11 months (mean 102.5 days). At follow-up angiography, we evaluated the fate of the injured arteries and the success rate of subsequent TACE.

Image evaluation

On DSA images, iatrogenic intimal injury was diagnosed when even one of the following findings was seen: (1) contrast agent remaining under the intima, (2) presence of double-barreled dissection, and (3) presence of extravasation [6].

Clinical variables were recorded on a standardized form, including information on patient demographics, history, clinical presentation, physical findings, imaging results, treatment, and outcomes (including mortality). Assessment of the DSA images and reports was done independently by two interventional radiologists with over 20 years of experience (E.S. and I.S.), with attention paid to the following: (a) presence and location of arterial injury; (b) length of arterial injury; (c) presence of extravasation; (d) presence of occlusion at the end of the procedure; and (e) sequential changes of arterial injury at follow-up. Final decisions regarding the classification of lesions were reached by consensus 
between the two radiologists.

\section{Statistical analysis}

Categorical variables are described as numbers and percentages, and were compared with the $x^{2}$ test or Fisher's exact test, as appropriate; the paired $t$-test and the Mann-Whitney U-test were used for continuous variables. In all analyses, $p<0.05$ was considered to indicate statistical significance. Analyses were performed with StatView J-5.0 software for Windows (Abacus Concepts, Berkeley, CA).

\section{Results}

Arterial injury during the TACE procedure

DSA findings of iatrogenic intimal injury were as follows: (1) contrast agent remaining under the intima $(n=31)$, (2) presence of double-barreled dissection $(n=6)$, and/or (3) presence of extravasation $(n=5)$. Extravasation occurred in five cases (Fig. 1). In four of these extravasation cases, embolization was performed using coils $(n=2)$ or gelatin sponge particles $(n=2)$. In the remaining one case, extravasation resolved without embolization. The sites of arterial injury were as follows: two in the celiac trunk, two in the proper hepatic artery, six in the left hepatic artery, 14 in the right hepatic artery, two in the gastroepiploic artery, eight in the right inferior phrenic artery, one in the lumbar artery, one in the left gastric artery, one in the celiac trunk to the common hepatic artery, and one in the proper hepatic artery to the left hepatic artery. Fifteen cases (39.5\%) of iatrogenic arterial injury were cases in which a 
replaced hepatic artery or an extrahepatic artery was the parasitic supply (Fig. 2). In two patients, arterial injury occurred twice in the same place.

Six arterial injuries were considered long $(>3 \mathrm{~cm})$, and 17 arterial injuries occurred in a proximal artery (proximal to the segmental artery).

In 34 of 38 cases ( $89.5 \%)$, arterial injury was caused by 0.016 -inch guide wire using the microcatheter. The remaining four arterial injuries were caused by 0.035 -inch steerable guide wire.

TACE was successful in 33 of 38 cases (86.8\%). In five cases, TACE was unsuccessful due to complete obstruction of the feeding artery $(n=3)$ or extravasation $(n=2)$.

Follow-up angiography showed remaining arterial stenosis in $81.6 \%$ (31 of 38 cases), and complete obstruction in $13.2 \%$ (five of 38 cases) at the end of the procedure. In the remaining two cases, final angiography showed no stenosis at the site of injury at the end of the procedure.

There were no cases with liver failure after TACE.

\section{Follow-up angiography}

In 36 of 38 cases (94.7\%), follow-up angiography (TACE or transcatheter arterial infusion) was performed within 1 year (range 1-11 months, mean 4.3 months). In 33 of 36 cases, the injured artery was patent; therefore, the recanalization rate was $91.7 \%$. In 24 cases, the injured artery was normalized without stenosis. In nine cases, stenosis remained. In one of the nine cases with persistent stenosis, both arterial stenosis and dilatation were seen due to double-barreled dissection. There were no cases with saccular aneurysm 
(pseudoaneurysm) formation. In three cases, the site of injury was obstructed; however, in two of these three cases, coil embolization of the injured artery had been performed because of extravasation.

We divided 36 cases into two groups: complete reopening $(n=24)$ and noncomplete reopening (stenosis or occlusion; $n=12$ ). Comparing the two groups, we evaluated the risk factors for non-complete reopening of the injured artery at follow-up. Injury length $>3 \mathrm{~cm}(p=0.0002)$, proximal arterial injury (proximal to the segmental artery; $p=0.03$ ), and prothrombin time-international normalized ratio (PT-INR; $p=0.025$ ) were significant risk factors for non-complete reopening (Table 2).

Age, sex, Child-Pugh score, TACE session number, follow-up period (interval between sessions), etiology, presence of obstruction at the end of the procedure, presence of extravasation, injury site at the extrahepatic artery or replaced hepatic artery, presence of ascites, maximum tumor size $>5 \mathrm{~cm}$, number of tumors $>5$, unilobar tumor location, extrahepatic metastasis, platelet count, alpha-fetoprotein, protein induced by Vitamin $\mathrm{K}$ absence or antagonistsII, and activated partial thromboplastin time were not significant risk factors for non-complete reopening (Table 2).

\section{Discussion}

Earlier reports of iatrogenic intimal injury of the celiac or hepatic artery revealed an incidence of $0.5-2.7 \%$ during TACE $[5,6,9,10]$. However, these reports were conducted on relatively old TACE procedures in which large catheters were used. Few studies have investigated TACE procedures using 
microcatheters. In the present series, the incidence of arterial injury during TACE was $1.7 \%$, which is relatively low and similar to the results of previous reports $[5,6,9,10]$. In addition, 34 cases of arterial injury were caused by 0.016-inch guide wire. Despite improvements in tools, the incidence of arterial injury has not improved. Recently, challenging TACE cases have increasingly been performed using an advanced microcatheter and microguidewire system. This may be why the incidence of arterial injury has not decreased despite the progress in device technology.

In the present study, arterial injury frequently occurred in the extrahepatic artery or the replaced hepatic artery. With the development of the TACE procedure, the number of cases undergoing repeated TACE procedures has increased, and the incidence of TACE through extrahepatic collateral vessels has also increased. In such cases, the TACE procedure is potentially more difficult than usual, as the collateral vessels are usually tortuous and smallsized. Therefore, arterial injury is more likely to occur in the extrahepatic artery or the replaced hepatic artery than the normal hepatic artery.

In two patients, arterial injury occurred twice at the same place because the artery might be weakened by previous injury. Cases in which TACE must be performed at a site where damage has previously occurred require careful catheterization.

The present series included five cases with extravasation. In two of the five cases, coil embolization was done to stop the bleeding, and gel (temporary embolic material) was used in two cases. At the subsequent TACE session, reTACE could not be successfully performed in two cases with complete 
obstruction due to coil embolization. Therefore, if arterial perforation occurs during TACE, the use of permanent embolic material should be avoided.

The recanalization rate in the present study $(91.7 \%)$ was higher than that reported in previous studies. Previous studies have reported recanalization rates of $71 \%$ and $64 \%$ in the celiac or hepatic artery after intimal injury [5, 6, 9]. Pseudoaneurysm formation after arterial injury was reported in several studies $[5-7,11,12]$; however, there were no cases with pseudoaneurysm formation in the present study. As the devices currently used are smaller than those used in previous studies, it is considered that arterial injury has become less severe. Although the frequency of arterial injury has not markedly decreased with improvement of the devices, complications associated with arterial injury seem to be milder.

In the present study, length of injury $>3 \mathrm{~cm}$ and proximal arterial injury (proximal to the segmental artery) were significant risk factors for non-complete reopening of the injured artery. All six cases with injury length $>3 \mathrm{~cm}$ also had proximal arterial injury. The reasons for these results are unknown. One potential reason may be that the proximal arteries have a relatively large diameter and thick arterial wall. Hence, the guidewire under the intima may easily progress into the long interval, and arterial injury at the proximal portion may tend to be long $(>3 \mathrm{~cm})$. Long arterial injuries tend to cause subsequent stenosis by damaging a wide range of arterial walls.

PT-INR was also a significant risk factor for non-complete reopening. PT-INR is an assay that evaluates the extrinsic pathway of coagulation. It is used to determine the clotting tendency of blood in the measure of warfarin dosage, 
liver damage, and vitamin $\mathrm{K}$ status [13]. Mean PT-INR was significantly longer in the complete reopening group than in the non-complete reopening group. The reason for this is unclear. A potential explanation is that reducing coagulability may avoid occlusion or stenosis of the injured arterial wall by thrombosis.

In the present study, the TACE session number was not a significant risk factor for non-complete reopening of the injured artery. However, TACE consists of selective catheterization and an intra-arterial infusion of a mixture of iodized oil or doxorubicin hydrochloride, cisplatin, and mitomycin followed by embolization with gel foam $[14,15]$. These procedures may mechanically traumatize the intimal layer of the arterial wall. The agents used may also cause contact damage to the intima and increase the vessel fragility [14]. Therefore, careful catheterization is needed in repeat TACE cases.

This study had the following limitations. First, the study was not prospective. The recorded descriptions of imaging findings and procedure might be insufficient. Second, the sample size was relatively small, and the follow-up period (interval until the subsequent TACE session) varied. Although the followup period was not a significant risk factor for non-complete reopening of the injured artery, further prospective studies on a larger scale are needed.

\section{Conclusions}

latrogenic arterial injury during the TACE procedure is a relatively rare complication. latrogenic arterial injury occurred more frequently in the extrahepatic artery or replaced hepatic artery (39.5\%). In such cases, careful 
catheterization is needed. The recanalization rate of injured arteries was high (91.7\%); however, length of injury $>3 \mathrm{~cm}$ and proximal arterial injury can be considered as risks for non-complete reopening of the injured artery.

\section{Ethical approval:}

For this type of study, formal consent is not required. 


\section{References}

1. Yamada R, Sato M, Kawabata H, Nakamura K, Takashima S

Hepatic artery embolization in 120 patients with unrespectable hepatoma.

Radiology 1983;148:397-401.

2. Lencioni R, Crocetti L.

Local-regional treatment of hepatocellular carcinoma.

Radiology. 2012;262:43-58.

3. Fidelman N, Kerlan RK.

Transarterial Chemoembolization and 90Y radioembolization for hepatocellular carcinoma: review of current applications beyond intermediate-stage disease.

AJR Am J Roentgenol 2015; 205:742-752

4. Bruix J, Sherman M; American Association for the Study of Liver Diseases. Management of hepatocellularcarci noma: an update.

Hepatology 2011; 53:1020-1022

5. Soo CS, Wallace S, Chuang VP, Charnsangavej C, Bowers TA

Injury to the intima of the hepatic artery: results of follow up in 11 cases.

Radiology 1982;143:373-378

6. Yoon DY, Park JH, Chung JW, Han JK, Han MC. latrogenic Dissection of the Celiac Artery and Its Branches During Transcatheter Arterial Embolization for Hepatocellular Carcinoma:Outcome in 40 Patients. Cardiovasc Intervent Radiol 1995:18:16-19.

7. Sakamoto I, Aso N, Nagaoki K, Matsuoka Y, Uetani M, Ashizawa K, Iwanaga

S, Mori M, Morikawa M, Fukuda T, Hayashi K, Matsunaga N. Complications associated with transcatheter arterial embolization for hepatic tumors. 
Radiographics. 1998;18:605-19

8. Feliciano DV. Evaluation and treatment of vascular injuries. In: Browner BD, Jupiter JB, Levine AM, Trafton PG, Krettek C, eds. Skeletal Trauma. Basic Science, Management, and Reconstruction. Philadelphia, PA: Saunders Elsevier; 2009:323-340.

9. Jonsson K, Lunderquist A, Pettersson $\mathrm{H}$, Sigstedt B. Subintimal injection of contrast medium as a complication of selective abdominal aortography. Acta Radiol 1977:18:55-64

10. Reuter SR. Development of collateral vessels in an acute occlusion of the common hepatic artery. AJR Am J Roentgenol 1966;97:473-6 11. Long JA, Krudy A, Dunnick NR, Cramer H, Schafer DF, Doppman JL, McCarthy DM. False aneurysm formation following arteriographic intimal dissection: Serial studies in 2 patients. Radiology 1980:135:323-6 12. Chuang VP, Wallace S, Stroehlein J, Yap HY, Part YZ. Hepatic artery infusion chemotherapy: Gastroduodenal complications. AJR Am J Roentgenol 1981;137:347-350.

13. Mallett SV, Chowdary P, Burroughs AK.

Clinical utility of viscoelastic tests of coagulation in patients with liver disease. Liver Int. 2013: 33: 961-974

14. Lin TS, Chiang YC, Chen CL, Concejero AM, Cheng YF, Wang CC, Wang SH, Liu YW, Yang $\mathrm{CH}$, Yong CC.

Intimal dissection of the hepatic artery following transarterial embolization for hepatocellular carcinoma: an intraoperative problem in adult living donor liver transplantation. Liver Transpl. 2009;15:1553-6. 
15. Cheng Y, Kan Z, Chen C, Huang T, Chen T, Yang B, Ko S, Lee T.

Efficacy and safety of preoperative lobar or segmental ablation via transarterial administration of ethiodol and ethanol mixture for treatment of hepatocellular carcinoma:clinical study. World J Surg 2000;24:844-850. 
Tables

Table 1. Clinical features and computed tomography findings

in 38 transcatheter arterial chemoembolization (TACE) sessions

Parameter $(n=38)$

Datum

Age, mean $\pm S D, y$

$71.8 \pm 11.2$

Female gender, $\mathrm{n}(\%)$

11(29)

Child-phug score, mean \pm SD

$5.8 \pm 0.9$

Session number of TACE, mean \pm SD

$2.2 \pm 1.8$

Etiology

Hepatitis B, n(\%)

12(32)

Hepatitis C, $n(\%)$

Alcohol, $n(\%)$

6(16)

Others, $\mathrm{n}(\%)$

6(16) 
CT findings

$\begin{array}{ll}\text { Presence of ascites, } \mathrm{n}(\%) & \text { 7(18) }\end{array}$

Maximum size of tumor $>5 \mathrm{~cm} \mathrm{( \% )} \quad$ 13(34)

Number of tumor $>5, \mathrm{n}(\%)$

Tumor location in unilobar, $\mathrm{n}(\%) \quad 9(24)$

Extrahepatic metastasis, $\mathrm{n}(\%)$

SD: standard deviation, CT: computed tomography 
Table 2 Factors associated with non-complete reopening of the injured artery

\begin{tabular}{|c|c|c|c|}
\hline Parameter $(n=38)$ & $\begin{array}{c}\text { Complete } \\
\text { reopening } \\
(n=24)\end{array}$ & $\begin{array}{c}\text { Non-complete } \\
\text { reopening } \\
(n=12)\end{array}$ & $P$ value \\
\hline Age, mean \pm SD, y & $74.4 \pm 9.6$ & $68.8 \pm 13.5$ & 0.1138 \\
\hline Female gender, $\mathrm{n}(\%)$ & $6(25)$ & $5(42)$ & 0.346 \\
\hline Child-phug score, mean \pm SD & $5.7 \pm 0.9$ & $6.0 \pm 1.0$ & 0.3481 \\
\hline Session number of TACE, mean \pm SD & $2.7 \pm 2.5$ & $2.0 \pm 1.2$ & 0.7413 \\
\hline Follow-up period (next session) (month), mean \pm SD & $3.0 \pm 2.2$ & $3.8 \pm 26$ & 0.3755 \\
\hline \multicolumn{4}{|l|}{ Etiology } \\
\hline Hepatitis B, $n(\%)$ & $7(29)$ & $3(25)$ & 0.7338 \\
\hline Hepatitis C, $n(\%)$ & $11(46)$ & $5(42)$ & 0.9181 \\
\hline Alcohol, $\mathrm{n}(\%)$ & 2(8) & $2(17)$ & 0.2309 \\
\hline Others, n(\%) & $4(17)$ & $2(17)$ & 0.6705 \\
\hline \multicolumn{4}{|l|}{ Angiographic findings of arterial injury } \\
\hline Long length of injury (>3cm), $n(\%)$ & $0(0)$ & $6(50)$ & 0.0002 \\
\hline Site of proximal hepatic artery, $\mathrm{n}(\%)$ & $8(33)$ & $9(75)$ & 0.012 \\
\hline Presence of obstruction at end of procedure, $\mathrm{n}(\%)$ & $3(13)$ & $2(17)$ & 0.2155 \\
\hline Presence of extravasation, $\mathrm{n}(\%)$ & $3(13)$ & $2(17)$ & 0.2166 \\
\hline Site of extrahepatic artery or replaced $\mathrm{HA}, \mathrm{n}(\%)$ & $4(17)$ & $2(17)$ & 0.5064 \\
\hline \multicolumn{4}{|l|}{ CT findings before TACE } \\
\hline Presence of ascites, $\mathrm{n}(\%)$ & $4(17)$ & $3(25)$ & 0.5932 \\
\hline Maximum size of tumor>5cm (\%) & $3(13)$ & $3(25)$ & 0.373 \\
\hline Number of tumor $>5, \mathrm{n}(\%)$ & $6(25)$ & $6(50)$ & 0.1572 \\
\hline Tumor location in unilobar, $\mathrm{n}(\%)$ & $17(71)$ & $10(43)$ & 0.5208 \\
\hline Extrahepatic metastasis, $\mathrm{n}(\%)$ & $4(17)$ & $0(0)$ & 0.1248 \\
\hline
\end{tabular}


Labo data at TACE

$\begin{array}{lccr}\text { Platelet }\left(\times 10^{3} / \mu \mathrm{l}\right) & 126.8 \pm 58.4 & 138.8 \pm 105.0 & 0.7413 \\ \text { AFP }(>400 \mathrm{ng} / \mathrm{dL}), \mathrm{n}(\%) & 8(33) & 6(50) & 0.3831 \\ \text { PIVKA II }(>400 \mathrm{mAU} / \mathrm{ml}), \mathrm{n}(\%) & 14(58) & 6(50) & 0.5374 \\ \text { APTT }(\mathrm{sec}) & 41.2 \pm 21.1 & 56.3 \pm 21.1 & 0.2122 \\ \text { PT- INR } & 1.36 \pm 0.56 & 1.29 \pm 0.29 & 0.025\end{array}$

SD: standard deviation, CT: computed tomography, TACE: transcatheter arterial chemoembolization, HA: hepatic artery, AFP: alpha-fetoprotein, PIVKA II: protein induced by vitamin $\mathrm{K}$ absence or antagonists-II,

APTT: activated partial thromboplastin time, PT-INR: prothrombin time-international normalized ratio 


\section{Figure legends}

Fig. 1-74-year-old man with hepatocellular carcinoma.

A. Digital subtraction angiography (DSA) image shows tumor stain in the right lobe (arrow).

B. Angiogram shows dissection of the right hepatic artery (arrows).

C. DSA image taken 40 minutes after the arterial injury shows that diffuse stenosis remains (arrow).

D. DSA image taken 4 months after the initial procedure shows that the injured artery has almost normalized (arrow).

Fig. 2-72-year-old man with hepatocellular carcinoma.

A. Digital subtraction angiography (DSA) image showing the tumor stain with a branch of the gastroepiploic artery as the parasitic supply (arrow).

B. DSA image showing that the intimal injury was caused by the microguide wire (arrows).

C. DSA image taken 2 months after the initial procedure showing that the injured artery has almost normalized. 
Fig.1.

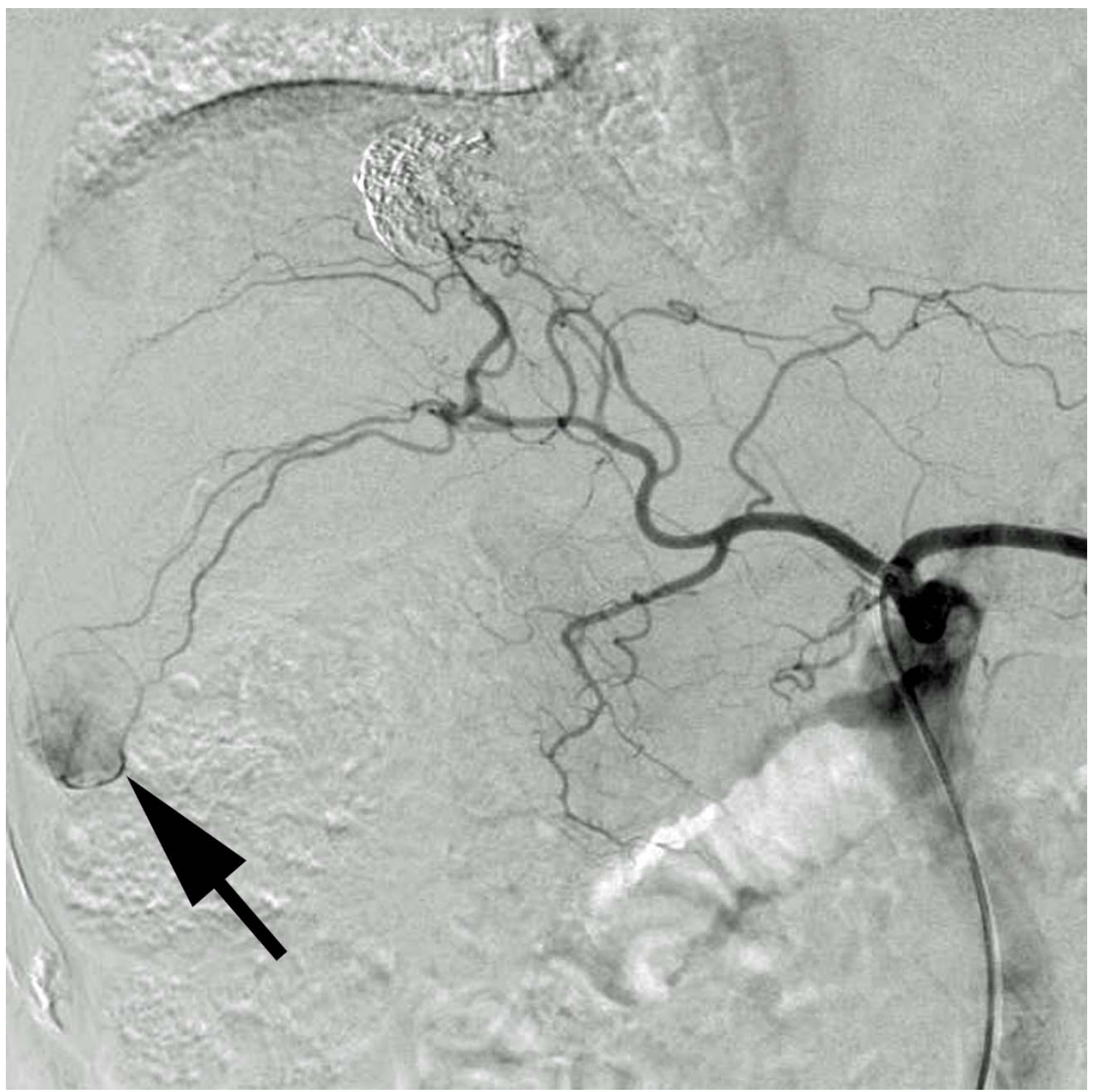




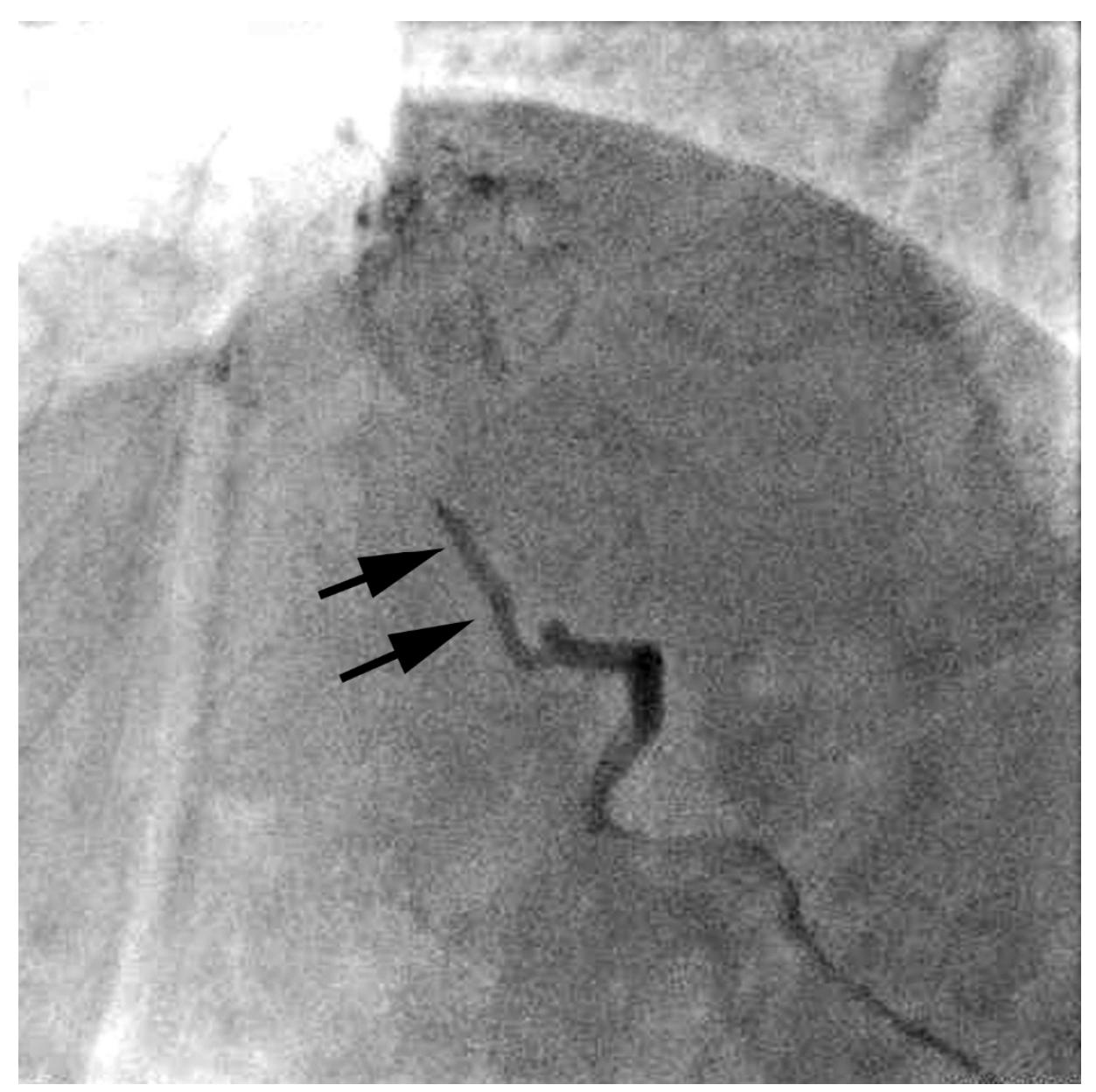




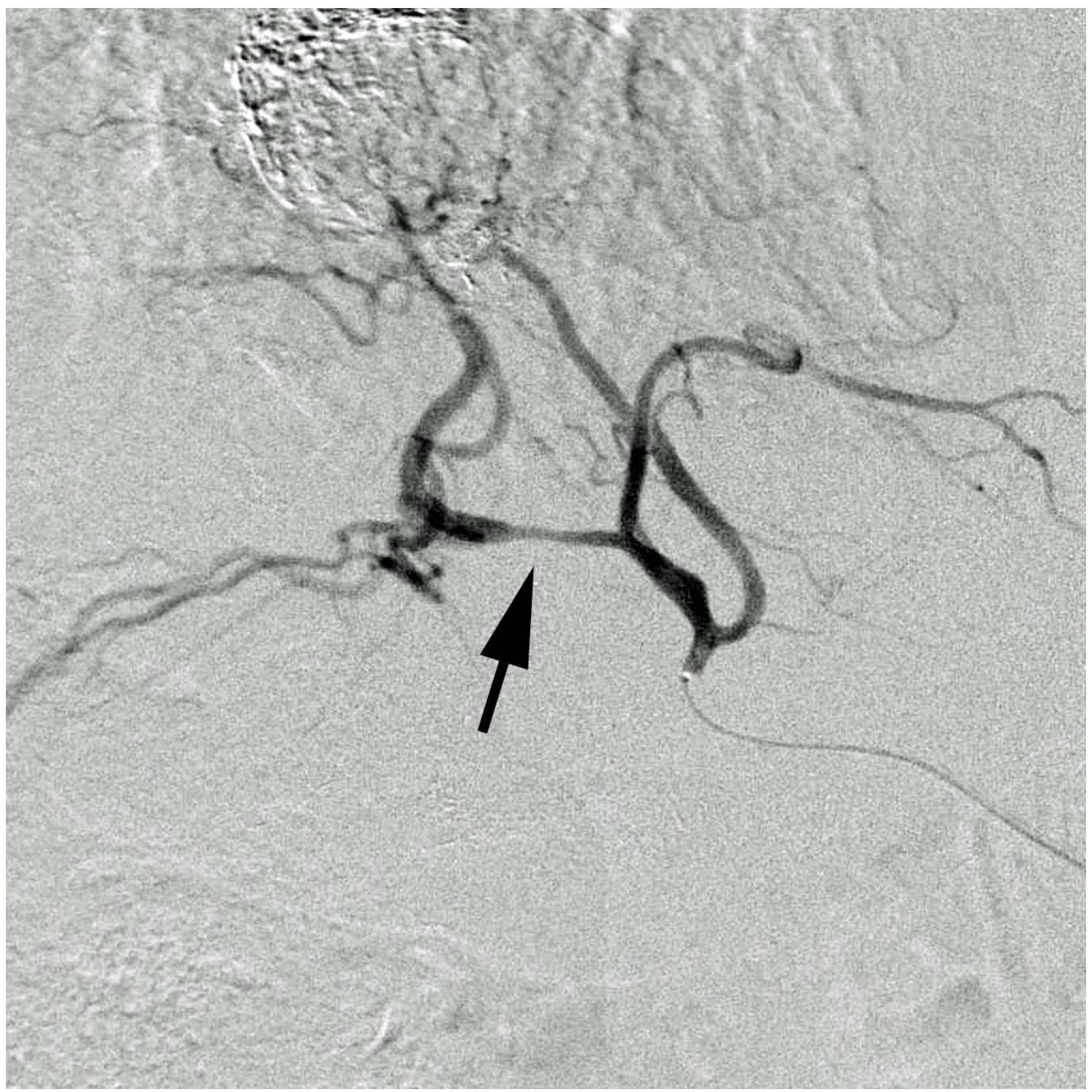




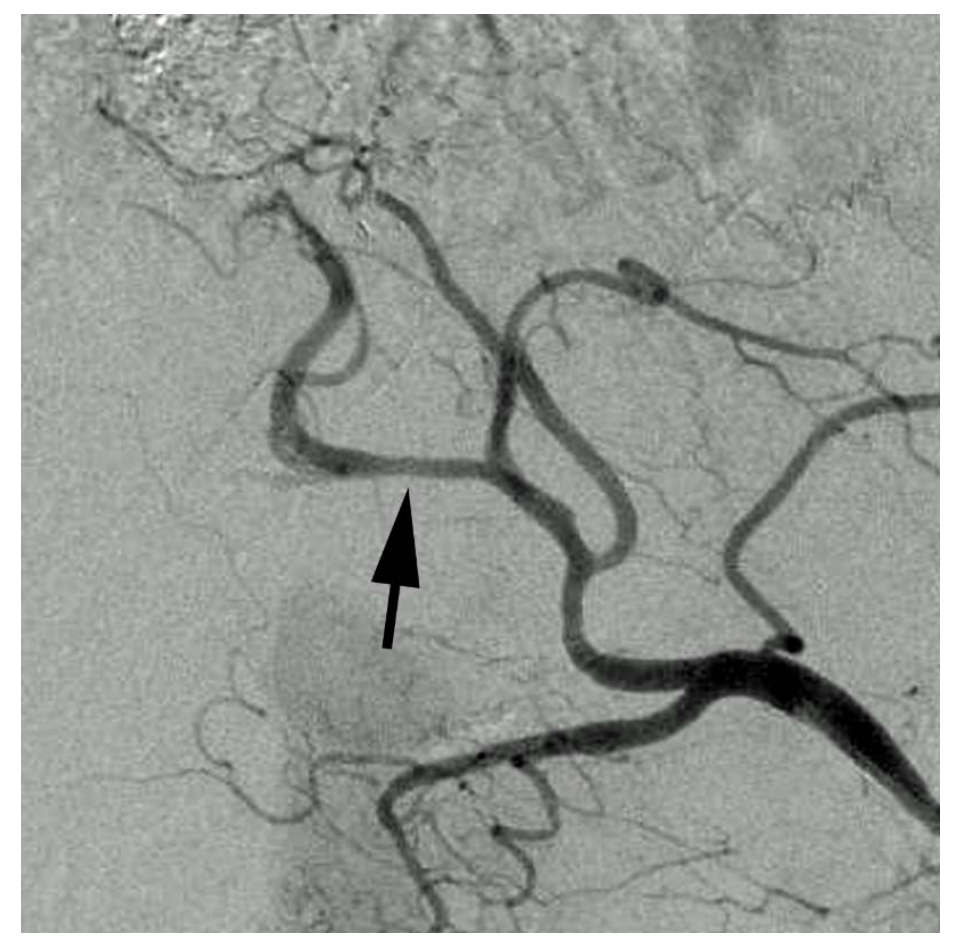


Fig.2
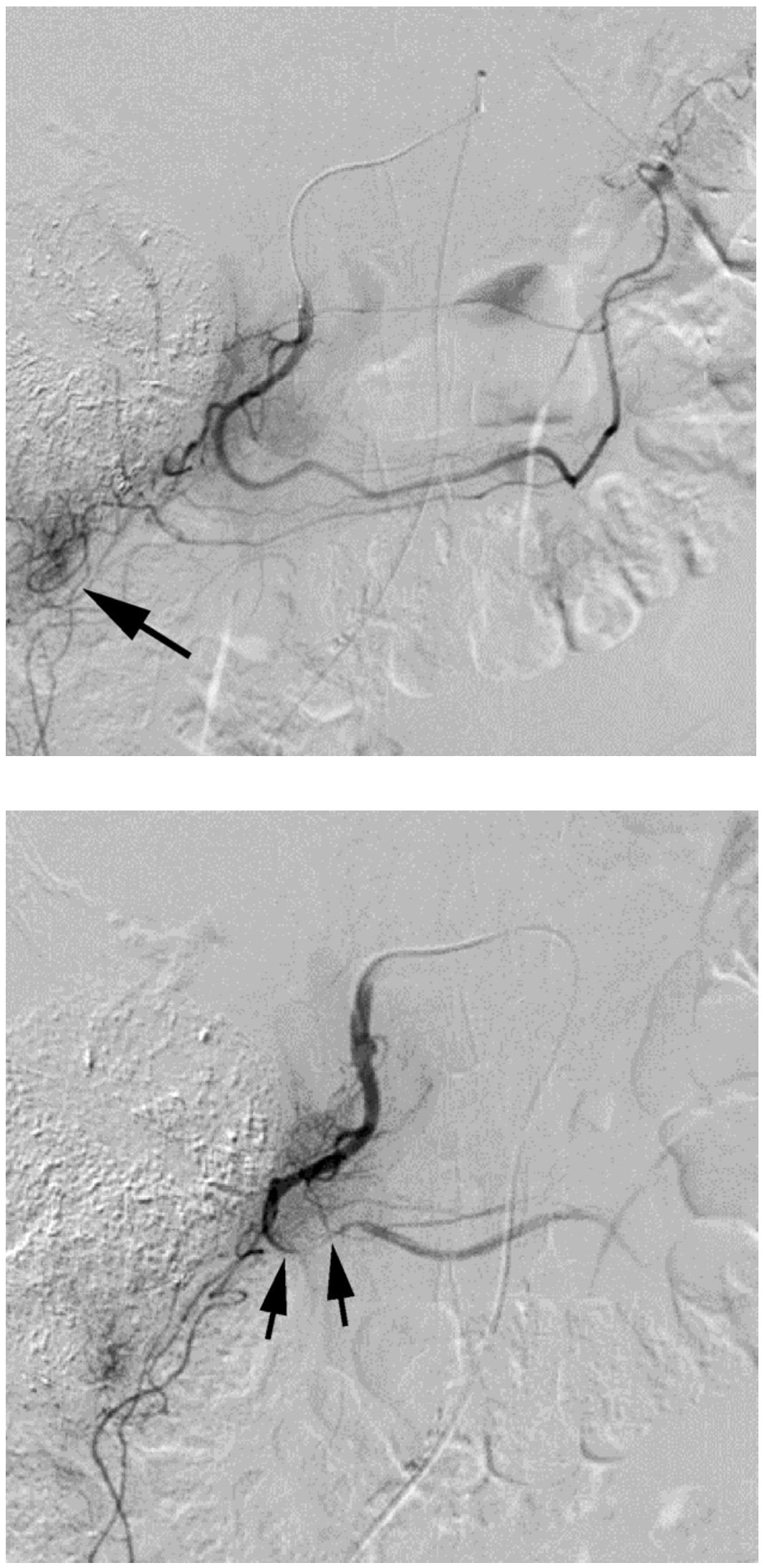


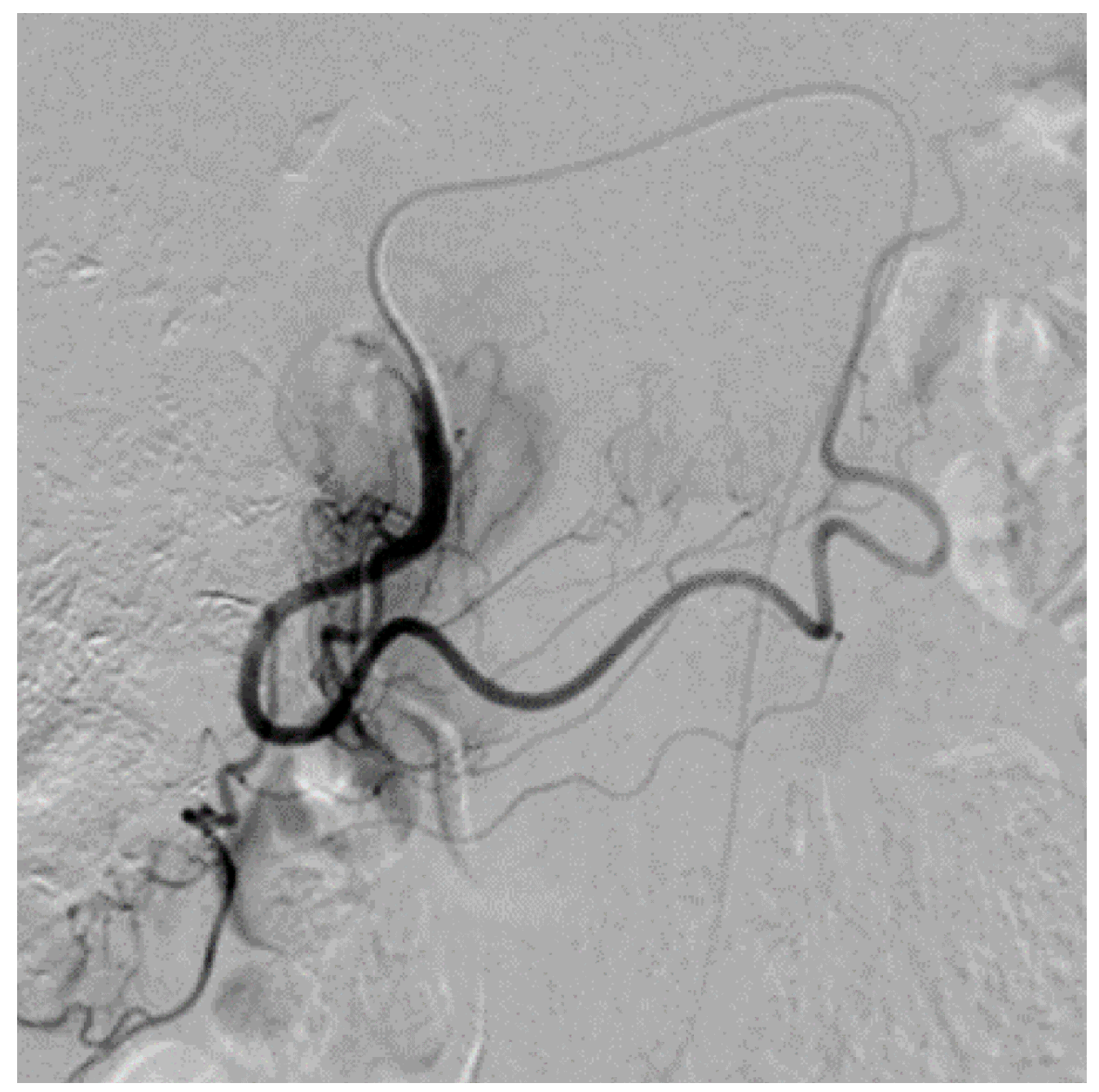

